\title{
Simulation of the Electron Transfer between the Tetraheme Subunit and the Special Pair of the Photosynthetic Reaction Center Using a Microstate Description
}

\author{
Torsten Becker, R. Thomas Ullmann, and G. Matthias Ullmann* \\ Structural Biology/Bioinformatics, University of Bayreuth, Universitätsstrasse 30, \\ BGI,95447 Bayreuth, Germany
}

Received: September 25, 2006; In Final Form: December 14, 2006

\begin{abstract}
Charge transfer through biological macromolecules is essential for many biological processes such as, for instance, photosynthesis and respiration. Protons or electrons are transferred between titratable residues or redox-active cofactors, respectively. Transfer rates between these sites depend on the current charge configuration of neighboring sites. Here, we formulate the kinetics of charge-transfer systems in a microstate formalism. A unique transfer rate constant can be assigned to the interconversion of microstates. Mutual interactions between sites participating in the transfer reactions are naturally taken into account. The formalism is applied to the kinetics of electron transfer in the tetraheme subunit and the special pair of the reaction center of Blastochloris viridis. It is shown that continuum electrostatic calculations can be used in combination with an existing empirical rate law to obtain electron-transfer rate constants. The re-reduction kinetics of the photo-oxidized special pair simulated in a microstate formalism is shown to be in good agreement with experimental data. A flux analysis is used to follow the individual electron-transfer steps.
\end{abstract}

\section{Introduction}

Electron transfer, often coupled to proton transfer, is one of the fundamental processes of biochemistry. In photosynthesis or oxidative phosphorylation, for instance, the transfer of electrons along a chain of redox-active sites enables biochemical systems to convert light or energy stored in chemical compounds into energy forms that can be used for other biochemical reactions. Impressive progress has been made over the last decades in understanding the underlying processes even on an atomic level. This progress was supported by the availability of structures of the involved proteins. ${ }^{1,2}$ The structural details allow to interpret data obtained by titration and kinetic experiments in terms of redox-active groups and transfer events between them. This detailed analysis gives a first idea about the microscopic picture of the biological function of these proteins. ${ }^{3}$ Available structures also enable computational investigation of these proteins, thus providing a theoretical insight into their molecular mechanism. Transfer processes require the knowledge of the equilibrium energetics of the involved states and the barriers connecting them. According to Marcus theory, the barrier determining a given electron-transfer rate depends on the free-energy difference between the donor state and the acceptor state, on the response of the surrounding media to changes of the charge distribution, the so-called reorganization energy, and on the electronic coupling between the donor state and the acceptor state. ${ }^{4,5}$ Empirical models based on Marcus theory proved to be very successful in reproducing electrontransfer rates for various biological electron-transfer systems. ${ }^{6-9}$ For systems with well-known equilibrium energetics, even simulations on complete electron-transfer systems have been performed. ${ }^{10,11}$ In those simulations, data not directly accessible

* Author to whom correspondence should be addressed. Fax: +49-92155-3544. E-mail: Matthias.Ullmann@uni-bayreuth.de. to experiment, such as the reorganization energies or interactions between redox-active groups, have been estimated from kinetic experiments. Electrostatic calculations have been shown to provide good estimates of redox potentials and of the interactions among sites in a protein ${ }^{12,13}$ as well as estimates of the reorganization energy. ${ }^{14}$ These quantities are required to calculate electron-transfer rates. To gain theoretical insight into transfer processes, it is desirable to simulate electron-transfer systems without referring to system-specific data derived experimentally.

It is known from experiments and theoretical calculations that the mutual interactions between sites of the system are crucial for an understanding of equilibrium and kinetic properties. ${ }^{15-17}$ In theoretical calculations of protonation and redox properties of proteins, these mutual interactions are reflected by describing the system in terms of microstates, i.e., a state of the system where every protonatable or redox-active site has a well-defined charge form. ${ }^{13}$ Titration properties for single sites are given by thermal averaging of the contribution of these microstates. Kinetic simulations, in contrast, commonly refer to transition events between individual sites. ${ }^{11,18}$ Although intuitive, such a description has a major drawback since, in general, it is not possible to assign a unique rate constant to a charge-transfer reaction between two given sites. The charge configuration of the surrounding sites may significantly influence the chargetransfer rate constant. Thus, in principle, there are as many charge-transfer rate constants between two given sites as there are charge configurations of neighboring sites. In the present work, we formulate kinetic equations of a transfer system in terms of the microstates already known from titration calculations. This approach resolves the ambiguities for calculating rate constants between sites. The rate constants are calculated using an empirical rate law. ${ }^{8}$ All energetic parameters required for rate calculations are obtained from electrostatic calculations. 
To test the accuracy of this theoretical approach, we simulate the electron-transfer kinetics of the multiheme subunit of bacterial photosynthetic reaction center (RC) and the so-called special pair (SP): a chlorophyll dimer.

The RC is an integral membrane protein, which couples the oxidation of a soluble cytochrome $\mathrm{c}$ or an iron sulfur protein on the periplasmic side of the membrane to the reduction of a quinone at the cytoplasmic side. ${ }^{3,19}$ Structures are available for various systems, ${ }^{20-22}$ and the equilibrium energetics as well as transfer kinetics have been accessed in numerous studies. ${ }^{23-26}$ The core of all reaction centers is formed by three subunits labeled H, M, and L. ${ }^{3}$ Following the photoinduced excitation of the SP an electron is transferred via several redox cofactors to the quinone $Q_{\mathrm{B}}$. Re-reduction of the $\mathrm{SP}$, i.e., the reduction after the photo-oxidation, is facilitated either directly by a soluble protein transport protein (e.g., Rhodobacter sphaeroides) or via an additional $\mathrm{C}$-subunit (e.g., Blastochloris viridis). The Csubunit, if present, contains four heme cofactors forming a transfer chain along the membrane normal. Electrons enter the C-subunit via a diffusing electron transport protein, which probably binds close to the outermost heme group. ${ }^{27}$

Photoinduced oxidation of the SP offers a well-defined initialization that can be controlled experimentally. Light absorption at various frequencies allows to follow the changes in the redox form of various cofactors. Electron transfer in the C-subunit is, thus, a process well-suited for kinetic analysis. Consequently, this subsystem was probed not only by equilibrium titration ${ }^{23,24}$ but also by kinetic experiments. ${ }^{25,28}$ With decreasing distance from the SP, the midpoint potentials of the hemes show a peculiar low-high-low-high pattern. Initial preparation of the system in various redox forms revealed a strong dependence of the kinetics of the re-reduction of the SP on the initial state of the system. As shown in ref 25, the reduction of the SP is the slowest if only the highest potential heme is reduced (heme $\mathrm{c}_{559}$ ). The re-reduction of the SP becomes faster upon reducing additional hemes and is 2 times faster if the three highest potential hemes are reduced. Since there is strong evidence that the re-reduction of the SP always occurs from heme $\mathrm{c}_{559},{ }^{28}$ this reaction is one example where charge-transfer rates between given sites depend significantly on the charges of their neighboring sites. An analysis using experimental redox potentials in combination with calculated interaction energies strongly suggests that the electrostatic interaction between heme $c_{559}$ and heme $c_{552}$ is responsible for the 2 -fold increase in the rate. ${ }^{28}$

In this article, we present a coherent scheme to simulate complex charge-transfer reactions in biological systems using coupled differential equations. Charge transfer is described as a transition from one microstate of the transfer system to another. Mutual interactions between sites are naturally taken into account within the presented formalism. Unique transfer rate constants can be assigned to transitions between microstates. Electron-transfer rates are estimated in the framework of Marcus theory. Equilibrium electrostatic calculations are used to access the energetics and mutual interactions between sites. The formalism is applied to electron transfer between the four heme cofactors and the SP of the $\mathrm{RC}$ of $B$. viridis. The simulated re-reduction kinetics of the photo-oxidized SP are in good agreement with experimental data.

\section{Theory}

It is common to describe charge transfer in proteins as a series of transfer events between groups involved in the transfer reaction. ${ }^{11,18}$ For example in electron transfer between the
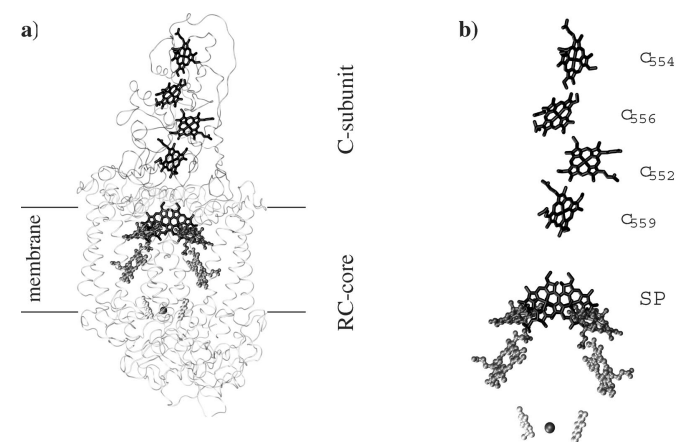

Figure 1. (a) Reaction center (RC) with the C-subunit of Blastochloris viridis. (b) Redox cofactors of the RC. The four hemes of the C-subunit form a near-linear transfer chain along the membrane normal. Electrons are transferred along this chain to reduce the special pair, SP.

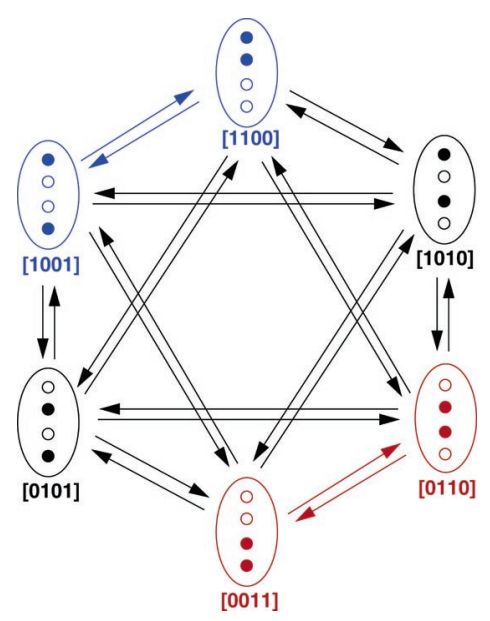

Figure 2. Charge-transfer system described by microstates, $\vec{x}=$ $\left(x_{1}, \ldots, x_{4}\right)$, where 1 or 0 denotes a reduced or oxidized site, respectively. The state transfer reactions colored blue and red represent charge transfer from site 4 to site 2, differing in the charge configuration of surrounding sites.

C-subunit and the SP of the RC, such a description corresponds to the attempt to estimate rate constants for the transfer between the heme cofactors and the SP directly. This approach, however, bears some problems as can be seen for the system depicted in Figure 2. This system has four redox-active sites and several possible transitions between them. The state of the system is described by a four-dimensional vector defining a microstate. The elements of this vector are either 1 or 0 , indicating whether the sites are reduced or oxidized, respectively. Each transition represents the transfer of an electron from one redox-active site to another. Examining, for example, the two transfer reactions [1001] $\rightarrow$ [1100] and [0011] $\rightarrow$ [0110] (blue and red in Figure 2, respectively), one can see that both events represent an electron transfer from site four to site two. Nevertheless, these transfer events are obviously not the same since they differ in the charge configuration of the other sites. Thus for the transfer from site four to site two, there are not one but several rate constants, depending on the number of possible configurations of the remaining sites. Figure 2, however, already suggests an alternative description of transfer reactions which will be outlined below.

System Description. Although, in general, no unique rate constant can be assigned to a charge transfer between two given sites of a multisite system, there is a unique transition between two given microstates. Thus, the ambiguities inherent for rates between individual sites can be resolved if transitions between 
microstates of the system are considered. A description based on microstates is already well established in theoretical calculations of equilibrium redox and protonation probabilities of proteins $^{12,13,29,30}$ and was proposed for charge-transfer systems by Bashford. ${ }^{31,32}$ Here, we use the concept of microstates to describe the kinetics of a charge-transfer system. To keep the notation simple, we formulate the theory for a system of redoxactive groups only, i.e., an electron-transfer system. Treating proton transfer or coupled systems is, however, straightforward.

We consider a system with $N$ redox-active sites. Each state of the system can be written as an $N$-dimensional vector $\vec{x}=$ $\left(x_{1}, \ldots, x_{N}\right)$, where $x_{i}$ is 0 or 1 if site $i$ is oxidized or reduced, respectively. A transfer event is described as a transition from one state vector to another. In the most general description, transitions are possible between any two given states. In practice, external constraints on the system may restrict the number of possible transitions. Such restrictions might concern the number of electrons in the system; i.e., if no electrons are allowed to enter or leave the system, then only transitions between state vectors with equal numbers of reduced sites will be allowed. If concerted transfer reactions cannot occur, then only transitions that represent a single electron transfer are considered. Such transfer events have the form $\left(x_{1}, \ldots, x_{i}, \ldots, x_{j}, \ldots, x_{n}\right) \rightarrow\left(x_{1}, \ldots\right.$, $\left.\tilde{x}_{i}, \ldots, \tilde{x}_{j}, \ldots, x_{n}\right)$ where $x_{i}$ and $\tilde{x}_{i}$ denote the redox state of site $i$ before and after the transfer event, respectively.

Each state of the system has a well-defined energy that depends on the energetics of the individual sites and the interaction between sites. The energy of a state $\vec{x}$ is given by ${ }^{33}$

$$
\begin{aligned}
G(\vec{x})=\sum_{i=1}^{N}\left(x_{i}-x_{i}^{g}\right) F\left(E_{i}^{\mathrm{intr}}\right. & -E) \\
& +\frac{1}{2} \sum_{i=1}^{N} \sum_{j=1}^{N}\left(x_{i}-x_{i}^{g}\right)\left(x_{j}-x_{i}^{g}\right) W_{i j}
\end{aligned}
$$

where $F$ is the Faraday constant, $x_{i}$ denotes the redox state of the site $i$ in state $\vec{x}, x_{i}^{\circ}$ is the reference form of site $i, E_{i}^{\text {intr }}$ is the redox potential that site $i$ would have if all other sites are in their reference form (intrinsic redox potential), $E$ is the reduction potential of the solution, and $W_{i j}$ represents the interaction of site $i$ with site $j$.

Equilibrium Properties. Equilibrium properties of a physical system are completely determined by the energies of its states. To keep the notation concise, states will be numbered by Greek indices; i.e., for state energies, $G_{v} \equiv G(\vec{x})$. For site indices, the roman letters $i$ and $j$ will be used.

The equilibrium probability of a single state is given by

$$
P_{v}^{\mathrm{eq}}=\frac{\mathrm{e}^{-\beta G_{v}}}{Z}
$$

with $\beta=1 / k T$ and $Z$ being the partition function of the system

$$
Z=\sum_{\nu=1}^{M} \mathrm{e}^{-\beta G_{v}}
$$

The sum runs over all $M$ possible states. Properties of single sites can be obtained from eq 2 by summing up the individual contributions of all states. For example, the probability of site $i$ being reduced is given by

$$
\left\langle x_{i}\right\rangle=\sum_{v}^{M} x_{v, i} P_{v}^{\mathrm{eq}}
$$

where $x_{v, i}$ denotes the redox form of site $i$ in the charge state $v$. For small systems, this sum can be evaluated explicitly. For larger systems, Monte Carlo techniques can be invoked to determine these probabilities.

For a system of interacting sites, the probabilities $\left\langle x_{i}\right\rangle$ can show a complex shape, thus rendering the assignment of midpoint potentials difficult or even meaningless. ${ }^{16}$ The energy differences between microstates, however, remain well-defined and thus form a convenient basis to describe the system.

Time Evolution. In the microstate description put forward in this article, charge-transfer events are described as transitions between well-defined microstates of a system. We simulate the time dependence of the population of each microstate using a master equation

$$
\frac{\mathrm{d}}{\mathrm{d} t} P_{\nu}(t)=\sum_{\mu=1}^{M} k_{\nu \mu} P_{\mu}(t)-\sum_{\mu=1}^{M} k_{\mu \nu} P_{\nu}(t)
$$

where $P_{v}(t)$ denotes the probability that the system is in charge state $v$ at time $t$ and $k_{v \mu}$ denotes the probability per unit time that the system will change its state from $\mu$ to $v$. The summation runs over all possible states $\mu$. Simulating charge transfer by eq 5 assumes that these processes can be described as a (stochastic) Markov process. This assumption implies that the probability of a given charge transfer only depends on the current state of the system and not on the way the system reached this state. Our approach excludes systems with strongly coupled microstates, e.g., delocalized charge systems, where the charge distribution can only be adequately described by a coherent superposition of various microstates. Delocalization of charges one mainly expects for strongly coupled electrontransfer systems due to the small mass of the electron. In biological systems, however, one often encounters long-range electron transfer, which is accurately described in the framework of Marcus theory. Thus, biological electron-transfer systems are well described in the nonadiabatic picture, i.e., in the weakcoupling limit.

Equation 5 is formally equivalent to kinetic rate equations known from physical chemistry. However, it should be noted that eq 5 describes the time evolution of the probability distribution of microstates of the system. For these microstates, as argued above, energies $G_{v}$ and transition probabilities $k_{\nu \mu}$ can be assigned unambiguously. The correct time-dependent probability of finding a single site in the reduced form can be obtained by summing up individual contributions from the timedependent probabilities $P_{\nu}(t)$

$$
\left\langle x_{i}\right\rangle(t)=\sum_{v}^{M} x_{v, i} P_{v}(t)
$$

Equation 5 is a coupled system of linear differential equations with constant coefficients. As explained in more detail in the Methods section, there is an analytical solution for nondegenerate systems that can be written as

$$
P_{v}(t)=\sum_{\mu}^{M} c_{\mu} v_{\mu, v} \mathrm{e}^{\alpha_{\mu} t}
$$

where $\alpha_{\mu}$ is the $\mu$ th eigenvalue of the matrix associated with eq $5, v_{\mu, v}$ is the $\nu$ th element of the $\mu$ th eigenvector of this matrix, and $c_{\mu}$ 's are integration constants determined from the initial condition of the system.

Flux Analysis. For analyzing a complex charge-transfer system, it is of particular interest to follow the flow of charges 
through the system, i.e., the charge flux. The flux from state $v$ to state $\mu$ is determined by the population of state $v$ times the probability per unit time that state $v$ will change into state $\mu$, i.e., by $k_{\mu \nu} P_{v}(t)$. The net flux between states $v$ and $\mu$ is thus given by

$$
J_{v \mu}(t)=k_{v \mu} P_{\mu}(t)-k_{\mu \nu} P_{v}(t)
$$

In the following, this flux between two states will be termed interstate flux. The interstate flux (eq 8) is positive if there is a net flux from state $\mu$ to state $\nu$.

In the common case, where the transition between $\nu$ and $\mu$ represents the transfer of a single charge between site $i$ and site $j$, this interstate flux corresponds to a flux between site $i$ and site $j, J_{i j}^{(\nu \mu)} \equiv J_{v \mu}$. The index $(\nu \mu)$ here indicates that this contribution to the total flux between sites $i$ and $j$ is related to the interstate flux $J_{v \mu}$. In general, there will be several interstate fluxes contributing to the charge transfer between sites $i$ and $j$. The total net flux between these sites $J_{i j}$ due to the interstate fluxes $J_{v \mu}$ is therefore given by the sum over interstate fluxes

$$
J_{i j}=\sum_{(\nu \mu)} J_{i j}^{(v \mu)}=\sum_{(\nu \mu)} J_{v \mu}
$$

Here the summation index $(v \mu)$ indicates that the sum has to be taken over all contributing interstate fluxes. The fluxes $J_{i j}$ will be referred to as intersite fluxes. Equipped with these elementary fluxes between pairs of sites, the complete flux network of the system can be deduced.

Calculation of the Rate Constants $\boldsymbol{k}_{\nu \mu}$. The outlined theory is directly applicable to a large class of reaction systems such as, for example, proton and electron transfer in proteins. The determination of the rate constants $k_{v u}$ will, however, be specific for the particular reactions that should be simulated. For electron-transfer systems, continuum electrostatic calculations in combination with existing empirical rate laws ${ }^{6,8}$ can be used to obtain electron-transfer rates in good agreement with experimental data. Three factors mainly govern the rate constants of biological electron-transfer reactions: the energy difference between the donor state and the acceptor state, the environmental polarization (reorganization energy), and the electronic coupling between the redox sites. The energy barrier for the transfer process is given in the framework of Marcus theory as

$$
\Delta G^{\ddagger}=\frac{\left(\Delta G^{\circ}+\lambda\right)^{2}}{4 \lambda}
$$

where $\Delta G^{\circ}$ is the energy difference between the donor state and the acceptor state and $\lambda$ is the so-called reorganization energy. The electronic coupling between the redox sites is commonly accounted for by a distance-dependent exponential function $A \exp \left(-\beta\left(R-R_{\mathrm{O}}\right)\right)$ where $R$ is the edge-to-edge distance between cofactors, $R_{\mathrm{o}}$ represents a Van der Waals contact distance, and $A$ represents an optimal rate. These aspects of biological electron transfer have been successfully combined to formulate a heuristic rate law applicable to long-range electron transfer ${ }^{6,8}$

$$
\begin{gathered}
\log \left(k_{\mathrm{ex}}\right)=13-0.6(R-3.6)-3.1 \frac{\left(\Delta G^{\circ}+\lambda\right)^{2}}{\lambda} \\
\log \left(k_{\mathrm{en}}\right)=13-0.6(R-3.6)-3.1 \frac{\left(-\Delta G^{\circ}+\lambda\right)^{2}}{\lambda}-\frac{\Delta G^{\circ}}{0.06}
\end{gathered}
$$

where $k_{\mathrm{ex}}$ and $k_{\mathrm{en}}$ are the rate constants for exothermic and endothermic electron-transfer reactions, respectively.

The free energy $\Delta G^{\circ}$ for a transition between two states $v$ and $\mu$ can be calculated within the electrostatic model using eq 1. The reorganization energy $\lambda$ contains two contributions, $\lambda=$ $\lambda_{\mathrm{o}}+\lambda_{i}$, where $\lambda_{\mathrm{o}}$ is the solvent reorganization energy and $\lambda_{i}$ accounts for changes of the nuclear degrees of freedom between the donor site and the acceptor site. $\lambda_{\mathrm{o}}$ was shown to be accessible to equilibrium calculations ${ }^{34}$ and formulated especially suited for Poisson-Boltzmann calculations ${ }^{14}$

$$
\lambda_{\mathrm{o}}=\frac{1}{2} \sum_{i}^{K}\left(\phi_{\mathrm{ad}}^{\mathrm{opt}}\left(\vec{r}_{i}\right)-\phi_{\mathrm{ad}}\left(\vec{r}_{i}\right)\right) \Delta q_{i}^{\mathrm{ad}}
$$

$\Delta q_{i}^{\text {ad }}$ is the change in charge of atom $i$ when going from the donor to the acceptor state. The potentials $\phi_{\mathrm{ad}}^{\mathrm{opt}}$ and $\phi_{\mathrm{ad}}$ are generated by the charge distribution $\Delta \rho=\rho_{\mathrm{a}}-\rho_{\mathrm{d}}$ in a low (opt) and a high dielectric environment, respectively. Here, $\rho_{\mathrm{a}}$ and $\rho_{\mathrm{d}}$ denote the charge distributions of the acceptor and donor states, respectively. The permittivity constant for the low dielectric environment reflects the electronic polarizability while the permittivity constant for the high dielectric environment accounts for the nuclear and electronic polarizabilities. The solvent reorganization energy is given by the difference in solvation free energies of the charge distribution $\Delta \rho$ between a low and a high dielectric environment. $\lambda_{i}$ 's can be estimated by density functional theory (DFT) calculations but are often found to be significantly smaller than the solvent reorganization energy. ${ }^{35-38}$ The inner sphere reorganization energy can be calculated from density functional theory as

$$
\lambda_{i}=E_{\text {bond }}\left(\vec{r}_{\mathrm{d}}, \rho_{\mathrm{a}}\right)-E_{\text {bond }}\left(\vec{r}_{\mathrm{a}}, \rho_{\mathrm{a}}\right)
$$

where $E_{\text {bond }}$ is the total DFT energy of the two sites and $\vec{r}_{\mathrm{d}}$ and $\vec{r}_{\mathrm{a}}$ correspond to the optimized geometries of the sites in the donor and acceptor states, respectively. Hence, the inner sphere reorganization energy is given by the difference in bonding energy between the donor and the acceptor geometries while the sites are kept in their acceptor state charge distribution. ${ }^{37,38}$

In this formulation, the total reorganization energy depends only on the charge difference between two states. Thus, the electron transfer between sites $i$ and $j$ is always connected with the same reorganization energy irrespective of the states that are converted into each other.

\section{Methods}

Structures and Parameters. In the calculations, we used the structure of the RC of Blastochloris viridis (PDB code 1PRC) ${ }^{39}$ having a resolution of $2.3 \AA$. Hydrogens were added with HBUILD ${ }^{40}$ in CHARMM ${ }^{41}$ and subsequently minimized using the CHARMM force field. ${ }^{42}$

The atomic partial charges for most atoms were taken from the CHARMM force field. The partial charges of the hemes, the special pair, and the linked residues were obtained from density functional calculations (functionals $\mathrm{VWN}^{43}$ and $\mathrm{PW} 91^{44}$ ) using the ADF program package. ${ }^{45}$ The electrostatic potentials obtained from the density functional calculation were fitted using the CHELPG algorithm ${ }^{46}$ combined with a singular value decomposition. ${ }^{47}$ The charges of all other cofactors are the same as those in previous calculations. ${ }^{48-50}$

Continuum Electrostatic Calculations and Equilibrium Redox Titration. All of the electrostatic calculations were done 
using the program suite MEAD, ${ }^{12,51}$ which uses a finite difference method to determine the electrostatic potential. For the calculations of the intrinsic redox potentials and the interaction energies, the dielectric constant of the protein and the dielectric constant of water were set to 4.0 and 80.0, respectively. The ionic strength was set to $0.1 \mathrm{M}$. For the protein, the electrostatic potential was calculated by focusing using three grids of $141^{3}, 121^{3}$, and $121^{3}$ grid points and grid spacings of of 2.0, 1.0, and $0.25 \AA$, respectively. For the model compounds, the electrostatic potential was calculated by focusing using two grids of $121^{3}$ grid points and grid spacings of of 1.0 and $0.25 \AA$. The first grid was centered on the protein or the model compound; the other grids were centered on the titratable group.

To calculate the energy of the different redox states, we first determined the protonation of the different protonatable residues in the protein using continuum electrostatics and Monte Carlo simulations. ${ }^{52} \mathrm{We}$ then fixed the highest populated protonation state to calculate the energy of the different redox states. The solution redox potentials of the special pair, of the bis-histidinyl heme, and of the metioninyl-histidinyl heme were adjusted to fit the equilibrium redox behavior as closely as possible. The equilibrium titration curves and the populations of the different redox states in dependence on the solution redox potential have been calculated by a statistical mechanics averaging.

Reorganization Energies. For the calculation of the solvent reorganization energy, we used a dielectric constant of 1.0 for the cofactor, 2.0 for the electronic dielectric constant, 4.0 for the total dielectric constant of the protein, and 80.0 for the water. In the calculation of the reaction field potential in the high dielectric environment, an ionic strength of $0.1 \mathrm{M}$ was considered. The electrostatic potential was calculated by focusing using three grids of $181^{3}, 181^{3}$, and $301^{3}$ grid points and grid spacings of of $2.0,1.0$, and $0.25 \AA$, respectively. The first grid was centered the on the geometric center of the protein; the other two grids were centered on the geometric centers of the cofactors between which the electron transfer takes place.

Inner sphere reorganization energies were computed from density functional calculations (functionals $\mathrm{VWN}^{43}$ and Becke Perdew $^{53}$ with a TZ2P basis set) using the ADF program. ${ }^{45}$ The interaction between the sites influences the inner sphere reorganization energy $\lambda_{i}$ only slightly. Therefore, $\lambda_{i}$ was calculated as the sum of the contributions of the two cofactors involved in the transfer reaction. ${ }^{38,54}$ The model sites consisted of the metal ions, the porphyrins, and the side chains axially coordinating the metal ions or binding the heme porphyrins. The amino acid side chains were cut at the $\mathrm{C}_{\alpha}$ atom, and their $\mathrm{C}_{\beta}$ atoms were fixed in their crystal structure positions. The heme propionates were cut off and substituted by hydrogen atoms. The phytyl tails of the special pair were truncated to methyl groups. Geometry optimizations with stringent convergence criteria were performed for each model site in its reduced and oxidized forms. Starting from the geometry-optimized structures, single-point calculations were performed to obtain the bonding energies of the model systems having the optimized geometry of one redox form and the respective opposite charge distribution.

Solution of the Differential Equation. The master equation in eq 5 can be rewritten as

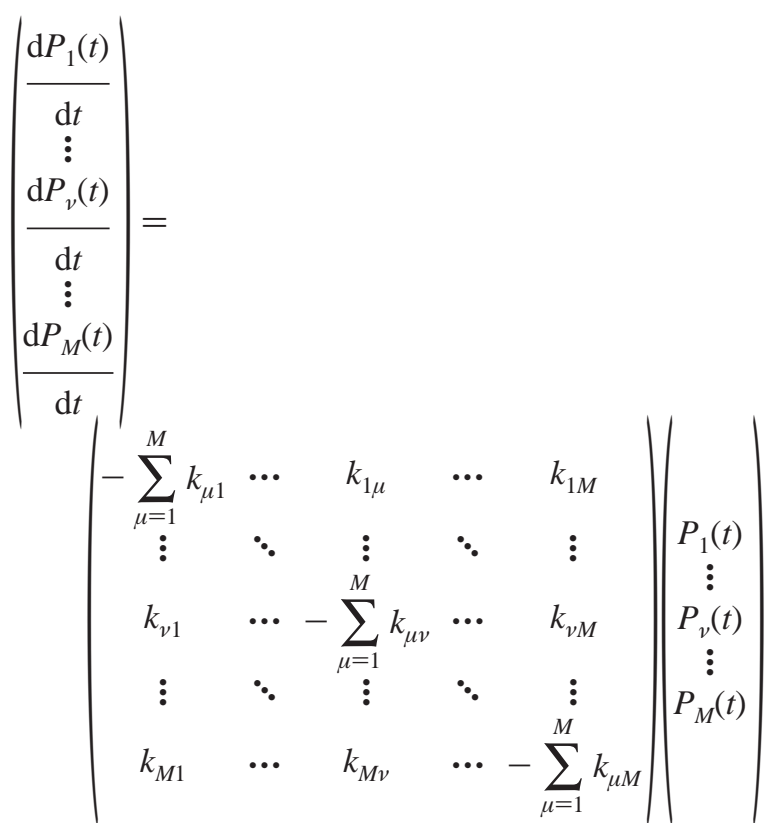

where $M$ is the number of states. For simplicity, we call the probability vector $\mathbf{p}$, the matrix of rate constants $\mathbf{A}$, and its elements $a_{\nu \mu}$; i.e., eq 14 becomes

$$
\frac{\mathrm{d} \mathbf{p}(t)}{\mathrm{d} t}=\mathbf{A p}(t)
$$

The diagonal elements $a_{v v}$ of matrix $\mathbf{A}$ are the negative of the sums over all of the rate constants $k_{\mu \nu}$ diminishing the population of state $v$. The diagonal elements $a_{v v}$, therefore, represent a decay rate for the population of state vector $\nu$. The off-diagonal element $a_{v \mu}$ is the rate constant $k_{v \mu}$ for the conversion of state $\mu$ to state $\nu$.

Equation 14 is a homogeneous system of first-order linear ordinary differential equations and can be solved formally as

$$
\mathbf{p}(t)=\exp (\mathbf{A} t) \mathbf{p}(0)
$$

A detailed balance criterium ensures that matrix $\mathbf{A}$ can be diagonalized, and if all eigenvalues are nondegenerate, then the solution can be written in the especially simple form

$$
\mathbf{p}(t)=\sum_{\mu=1}^{M} c_{\mu} \mathbf{v}_{\mu} \mathrm{e}^{\alpha_{\mu} t}
$$

where $\alpha_{\mu}$ and $\mathbf{v}_{\mu}$ are the eigenvalue and the corresponding eigenvector of matrix $\mathbf{A}$, respectively, and $c_{\mu}$ 's are constantsthat can be determined from the initial concentrations $\mathbf{p}$ at $t=0$ (i.e., all the terms $\mathrm{e}^{\alpha_{\mu} t}=1$ )

$$
\mathbf{p}(0)=\mathbf{V} \mathbf{c}
$$

where $\mathbf{V}$ is a matrix containing the eigenvectors of $\mathbf{A}$. For the general case of degenerate eigenvalues, eq 17 has to be slightly modified. ${ }^{55}$ For a more detailed discussion of master equations and their general behaviors, see, for example, refs 55 and 56.

To solve the system of differential equations in eq 14 in the form of eq 17, the eigenvalues and the eigenvectors of matrix A were determined using the routine DGEEV of the LAPACK library. ${ }^{57}$ The coefficients $c_{\mu}$ are determined by solving eq 18 using standard numerical methods (lower and upper triangular decomposition). ${ }^{58}$

\section{Results and Discussion}

In this article, we use a microstate formalism to simulate the kinetics of electron transfer between the C-subunit and the SP 
a)

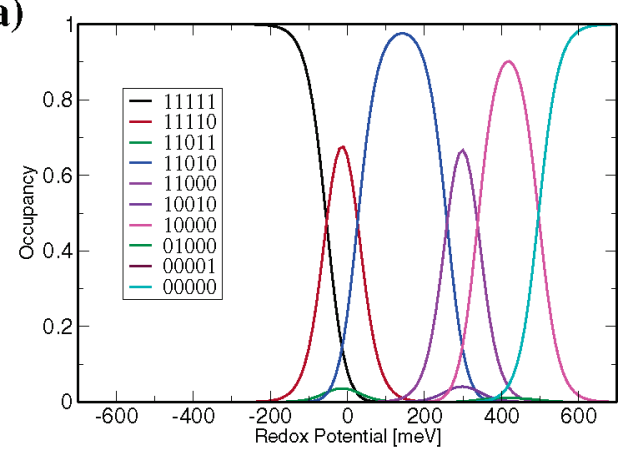

b)

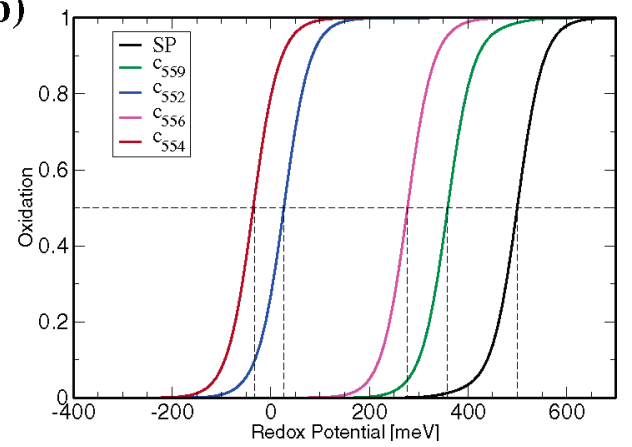

Figure 3. (a) Occupancies of significantly populated microstates, where 1 denotes reduced whereas 0 indicates the oxidized state. The state vector is given in the order $\left(\mathrm{SP}, \mathrm{c}_{559}, \mathrm{c}_{552}, \mathrm{c}_{556}, \mathrm{c}_{554}\right)$. (b) Titration curves for individual sites were calculated from eq 4 . The dashed lines indicate the midpoint potentials as measured in titration experiments.

TABLE 1: Calculated Equilibrium Midpoint Potentials Compared to Experimental Values

\begin{tabular}{|c|c|c|c|c|c|}
\hline$E_{\mathrm{m}}(\mathrm{meV})$ & SP & $\mathrm{c}_{559}$ & $\mathrm{c}_{552}$ & $\mathrm{c}_{556}$ & $\mathrm{c}_{554}$ \\
\hline calct & 500 & 359 & 27 & 278 & -35 \\
\hline Fritzsch et al. ${ }^{24}$ & 500 & 370 & 10 & 300 & -60 \\
\hline Dracheva et al. ${ }^{23}$ & 500 & 380 & 20 & 310 & -60 \\
\hline
\end{tabular}

of the RC. Poisson-Boltzmann electrostatics in combination with an empirical rate law provide transition probabilities between microstates. These rate constants are used to simulate the kinetic behavior of this system using coupled differential equations. The resulting re-reduction kinetics of the special pair SP reproduces the redox state dependence seen in experiments and are shown to be in quantitative agreement with the kinetic data obtained in ref. 25.

Midpoint Potentials. A prerequisite for a good description of the kinetics is to accurately account for equilibrium properties. Therefore, we compute first the equilibrium midpoint potentials for all four hemes and the special pair SP using PoissonBoltzmann electrostatics.

Figure 3 depicts the occupancies of those microstates that were found to be significantly populated. Experimentally observed Nernst-like titration curves are obtained for individual sites by summing up the contributions of these microstates as stated in eq 4. Table 1 lists the calculated midpoint potentials, which are compared to the experimental values. A good overall agreement is achieved; the characteristic high-low-high-low pattern with respect to the distance from the special pair is clearly reproduced, and the numerical values of all hemes are within the range of accuracy commonly achieved by electrostatic theory. ${ }^{59}$ The same intrinsic redox potentials and interaction energies that allow us to reproduce the redox-titration behavior of the RC are used to calculate microscopic redox potentials (Table 2). These microscopic redox potentials are required to compute rate constants.
TABLE 2: Intrinsic Redox Potentials and Interaction Energies for the SP and the Four Heme Groups of the C-Subunit of $B$. viridis ${ }^{a}$

\begin{tabular}{lrrrrrr}
\hline & $E^{\text {intr }}$ & SP & $\mathrm{c}_{559}$ & $\mathrm{c}_{552}$ & $\mathrm{c}_{556}$ & $\mathrm{c}_{554}$ \\
\hline $\mathrm{SP}$ & 451 & 0 & & & & \\
$\mathrm{c}_{559}$ & 286 & 39 & 0 & & & \\
$\mathrm{c}_{552}$ & 23 & 6 & 67 & 0 & & \\
$\mathrm{c}_{556}$ & 170 & 1 & 7 & 51 & 0 & \\
$\mathrm{c}_{554}$ & -36 & 0.1 & 1 & 5 & 58 & 0
\end{tabular}

${ }^{a}$ All values are given in $\mathrm{meV}$.

TABLE 3: Solvent and Inner Sphere Reorganization Energies and Cofactor Distances

\begin{tabular}{clccr}
\hline site 1 & site 2 & $\lambda_{\mathrm{o}}(\mathrm{eV})$ & $\lambda_{i}(\mathrm{eV})$ & $R(\AA)$ \\
\hline $\mathrm{SP}$ & $\mathrm{c}_{559}$ & 0.51 & 0.115 & 12.1 \\
$\mathrm{SP}$ & $\mathrm{c}_{552}$ & 0.58 & 0.100 & 24.6 \\
$\mathrm{SP}$ & $\mathrm{c}_{556}$ & 0.59 & 0.115 & 37.4 \\
$\mathrm{SP}$ & $\mathrm{c}_{554}$ & 0.59 & 0.115 & 50.9 \\
$\mathrm{c}_{559}$ & $\mathrm{c}_{552}$ & 0.44 & 0.063 & 6.9 \\
$\mathrm{c}_{559}$ & $\mathrm{c}_{556}$ & 0.57 & 0.076 & 21.5 \\
$\mathrm{c}_{559}$ & $\mathrm{c}_{554}$ & 0.59 & 0.076 & 35.4 \\
$\mathrm{c}_{552}$ & $\mathrm{c}_{556}$ & 0.46 & 0.063 & 8.1 \\
$\mathrm{c}_{552}$ & $\mathrm{c}_{554}$ & 0.57 & 0.063 & 21.5 \\
$\mathrm{c}_{556}$ & $\mathrm{c}_{554}$ & 0.41 & 0.076 & 7.1
\end{tabular}

Reorganization Energies. The next important parameter determining electron-transfer rate constants is the reorganization energy. Table 3 lists the calculated reorganization energies and the edge-to-edge distances between cofactors. The total reorganization energy $\lambda=\lambda_{\mathrm{o}}+\lambda_{i}$ varies between $0.48-0.66 \mathrm{eV}$. Experimental values for the reorganization energies for the electron transfer from the C-subunit to the SP and within the $\mathrm{C}$-subunit do not exist. Reorganization energies obtained on other biological systems have been reported in the range between 0.2 and $1.2 \mathrm{eV} . .^{14,60-62}$ Thus, although the reorganization energies obtained for our system cannot directly be compared to experimental data, the values obtained from our calculations are in agreement with literature values reported for biological transfer systems.

Kinetics. The midpoint potentials in Table 1 cannot directly be used to obtain the reaction free energy of a transfer event as necessary for eq 11. Midpoint potentials reflect the energetic cost to reduce a particular site while all sites are in equilibrium with the solution reduction potential. In contrast, in eq 11 the free energy for transferring an electron refers to the free-energy difference between two states differing in their charge configuration (microstate) by exactly the transferred electron. The latter energy can be directly related to a microscopic equilibrium constant, while the relation between a midpoint potential and a real equilibrium constant is not straightforward. ${ }^{16}$

To demonstrate that Poisson-Boltzmann electrostatics can be used in a microstate formalism to obtain valid descriptions of the kinetic properties of electron-transfer systems, we compare the re-reduction kinetics of the SP to the experimentally observed half-life of the oxidized state. ${ }^{25}$ Ortega et al. exposed the reaction center of $B$. viridis to different redox potentials, thus preparing the system in charge configurations with 4,3 , and 2 electrons distributed over the system consisting of the four hemes and the SP. The re-reduction kinetics of the SP were measured after photoinduced oxidation.

To mimic this experimental setup, four simulations were performed. First, all four hemes were set to their reduced forms, the SP was set to its oxidized form, and the kinetics of this system were simulated according to eq 7 . The kinetics of this first re-reduction reaction were not measured experimentally. The next three simulations started from the equilibrium distribu- 
States

a)

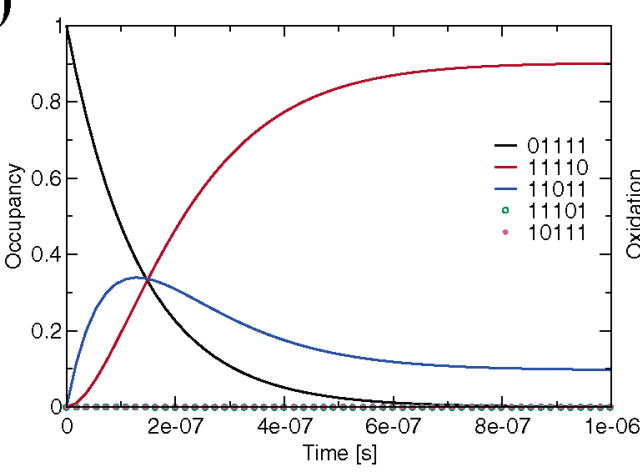

b)

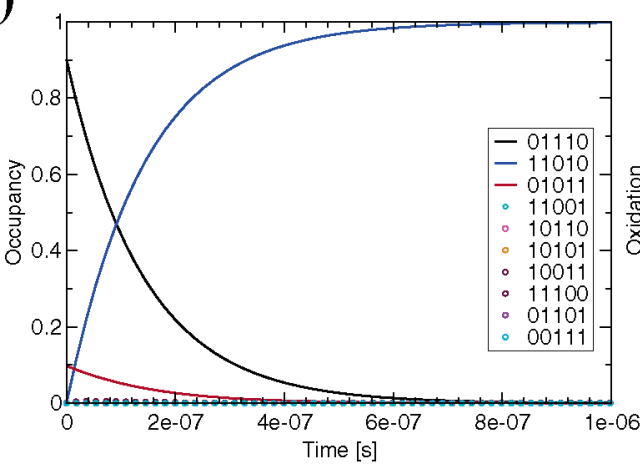

c)

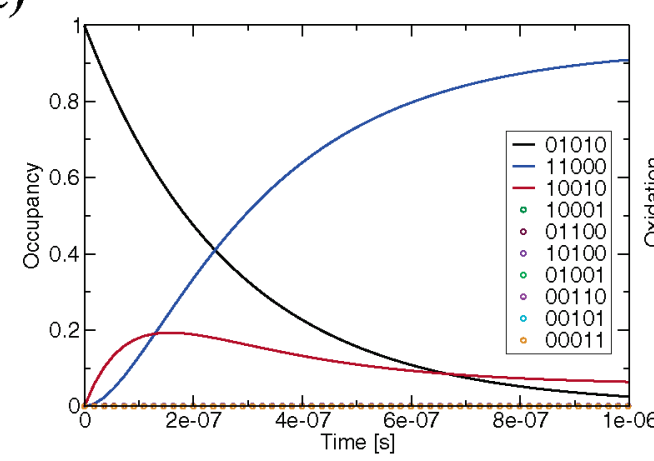

d)

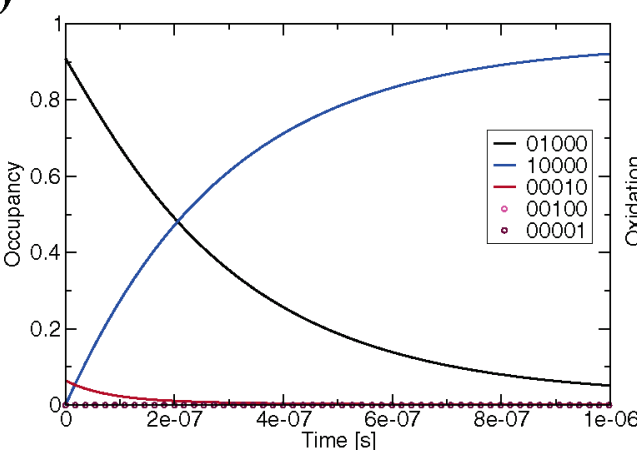

Sites
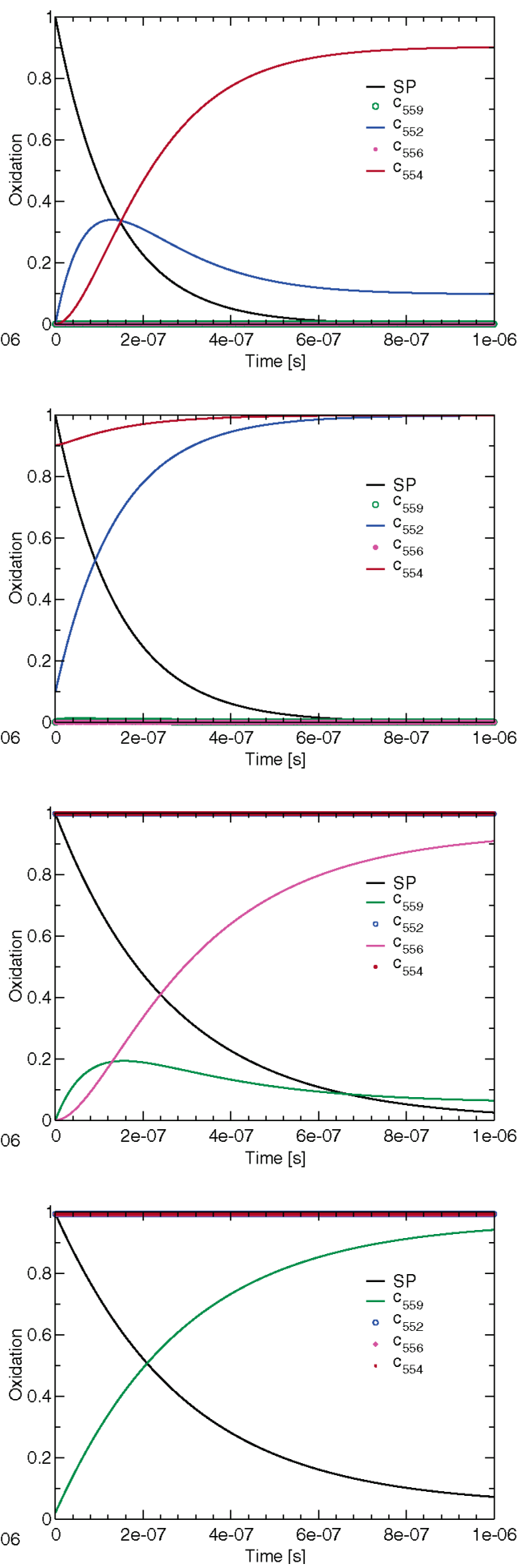

Figure 4. Left column depicting the time-dependent probability distribution of microstates after photo-oxidation of the SP simulated by eq 5. The state vector is given in the order $\left(\mathrm{SP}, \mathrm{c}_{559}, \mathrm{c}_{552}, \mathrm{c}_{556}, \mathrm{c}_{554}\right)$. The associated oxidation probabilities of the four hemes and the SP are depicted in the right column. Data shown in symbols do not significantly differ from either 0 or 1 . (a-d) The initial setup consists of 4, 3, 2, or 1 electrons distributed among the four hemes, respectively. Initial distributions for the microstates were taken from an equilibrium distribution prior to photo-oxidation of the SP.

tion of the previous run and setting the SP to its oxidized state, thus resembling a system with 4, 3, or 2 electrons present prior to instantaneous photo-oxidation of the SP.

An overview of the complete kinetics of the system is shown in Figure 4. Each state is characterized by a state vector where 1 denotes a reduced site and 0 an oxidized site. The order of sites is given from left to right: $\mathrm{SP}$, heme $\mathrm{c}_{559}$, heme $\mathrm{c}_{552}$, heme $\mathrm{c}_{556}$, and heme $\mathrm{c}_{554}$. This order corresponds to the spatial arrangement of the redox sites along the membrane normal. Figures $4 \mathrm{a}-\mathrm{d}$ correspond to simulations with $4,3,2$, and 1 electrons present after photo-oxidation of the SP, respectively. On the left side of Figure 4, the time-dependent probability 
TABLE 4: Half-Life of the Photo-oxidated State of the SP Dependent on the Number of Electrons in the System

\begin{tabular}{|c|c|c|c|c|}
\hline$t_{1 / 2}(\mathrm{~s})$ & 4 & 3 & 2 & 1 \\
\hline $\begin{array}{l}\text { experiment }{ }^{25} \\
\text { simulation }\end{array}$ & $93 \times 10^{-9}$ & $\begin{array}{r}115 \times 10^{-9} \\
99 \times 10^{-9}\end{array}$ & $\begin{array}{l}190 \times 10^{-9} \\
187 \times 10^{-9}\end{array}$ & $\begin{array}{l}230 \times 10^{-9} \\
210 \times 10^{-9}\end{array}$ \\
\hline
\end{tabular}

distribution of the accessible microstates is shown. The corresponding oxidation probabilities for the heme active sites and the SP are shown on the right side. In all simulations, it is observed that only a limited number of microstates contributes significantly to the probability distribution in the pico- to microsecond time scale.

To obtain half-lives for the oxidized state of the SP that can be compared to the experimental data, we performed an exponential fit on the re-reduction curves of the SP. For all our simulations a one-exponential fit was sufficient to obtain good agreement with the simulation data. The seemingly nonexponential character of the SP re-reduction in Figures $4 c$ and $4 d$ originates from the fact that the SP is not fully reduced in equilibrium; i.e., the re-reduction curve reaches a constant level above zero. This behavior was taken into account by fitting the re-reduction kinetics of the SP to the function

$$
f(t)=\frac{1}{1+a}\left(a+\mathrm{e}^{-\alpha t}\right)
$$

where $\alpha$ is the decay constant of the photo-oxidized form of the SP and $a /(1+a)$ represents its equilibrium probability. Table 4 lists the half-lives derived from this one-exponential fit of our simulation data compared to the half-lives of the very fast component obtained by Ortega et al. All values are within a factor of 1.5 compared to the experimental values. Furthermore, the increase of the lifetime upon decreasing the number of electrons in the system is clearly reproduced. This increase indicates that interactions among sites and therefore the interplay of the various possible charge states within the transfer system are reliably reproduced in the microstate formalism presented here. Thus, theoretical investigations on the basis of the theory outlined in this article promise to shed light on the function of more complicated reactions such as coupled transfer of protons and electrons.

Given the good agreement with experimental data, we further analyze the simulation by asking specific questions such as which microstates participate in the transfer process and which interactions are responsible for the observed changes in the kinetic behavior. In the present example, the simplicity of the system allows for straightforward answers of both questions within the present model. As was already suggested on the basis of electrostatic calculations in combination with experimental midpoint potentials, the change in redox kinetics upon reducing additional heme groups can be understood in terms of electrostatic interactions between the heme groups. ${ }^{28}$ Reduction of heme $c_{552}$ and heme $c_{556}$ destabilizes the reduced form of heme $\mathrm{c}_{559}$ and thus increases the transfer rate constants between heme $\mathrm{c}_{559}$ and the SP. Table 5 lists microscopic rates related to direct electron transfer from heme $\mathrm{c}_{559}$ to the SP. These rate constants only differ in the interactions between heme groups being in different redox forms. Since the transfer from heme $c_{559}$ to the $\mathrm{SP}$ is rate-determining for the reduction kinetics of the SP, they clearly indicate that the purely electrostatic interactions in the present model account for the experimentally observed dependence of lifetimes on the number of electrons in the system.

Flux Analysis. A picture of the individual transfer steps that contribute to the overall kinetic behavior can be obtained with a flux analysis as described in the Theory section. Figure 5
TABLE 5: Transfer Rate Constants and Associated Microscopic Half-Lives for State Transitions Representing Electron Transfer from Heme $c_{559}$ to the SP

\begin{tabular}{|c|c|c|c|c|}
\hline \multicolumn{2}{|c|}{ states } & \multirow[b]{2}{*}{$k_{\mu \nu}\left(\mathrm{s}^{-1}\right)$} & \multirow[b]{2}{*}{$t_{1 / 2}{ }^{\text {micro }}(\mathrm{s})$} & \multirow[b]{2}{*}{$t_{1 / 2}(s)$} \\
\hline donor & acceptor & & & \\
\hline 01111 & 10111 & $7.4 \times 10^{6}$ & $93 \times 10^{-9}$ & $93 \times 10^{-9}$ \\
\hline 01110 & 10110 & $7.4 \times 10^{6}$ & $93 \times 10^{-9}$ & $99 \times 10^{-9}$ \\
\hline 01010 & 10010 & $3.7 \times 10^{6}$ & $187 \times 10^{-9}$ & $187 \times 10^{-9}$ \\
\hline 01000 & 10000 & $3.5 \times 10^{6}$ & $198 \times 10^{-9}$ & $220 \times 10^{-9}$ \\
\hline
\end{tabular}

depicts the time-dependent fluxes for the simulations shown in Figure 4. Interstate fluxes shown in the left column are calculated from eq 8 , and the intersite fluxes in the right column are calculated from eq 9 . Only a limited number of interstate fluxes contribute significantly to the derived intersite fluxes, since only transfer events between next-neighbor sites contribute to the electron transfer between the four hemes and the SP on the sub-microsecond time scale. The next-neighbor constraint, in turn, is a consequence of the strong dependence of the electron-transfer rate constants on the cofactor distances as expressed in eq 11. This strong dependence on the cofactor distances led to the suggestion that these distances are the primary design factor in electron-transfer proteins. 8,9

Details of the transfer steps present in our simulations are depicted in Figure 6. For each of the four simulations, all possible microstates are depicted (Figures $4 \mathrm{a}-\mathrm{d}$ ). Filled and open circles denote the reduced and oxidized forms of a redox site, respectively. The order of the microstates is given from top to bottom as heme $\mathrm{c}_{554}$, heme $\mathrm{c}_{556}$, heme $\mathrm{c}_{552}$, heme $\mathrm{c}_{559}$, and the SP. The microstates depicted in the uppermost rows of each graph $a-d$ represent the starting configurations of the simulations. The microstates shown in the bottom rows represent the populations of microstates present at the end of the simulations. Only microstates contributing more than $0.1 \%$ to the starting or end configurations are considered. The row(s) in between the top and the bottom rows show all other possible microstates of the simulation. For the starting and final microstates the starting and final probabilities (in \%) are given in parentheses, respectively. For intermediate states, the values in parentheses denote the maximal probability observed during the simulation. Fluxes contributing significantly are indicated by arrows, and their maximum values are given. Each arrow indicates a net transition from one microstate to another, i.e., from one charge configuration of the system to another. As explained in detail, such a transition represents a transfer of an electron from one redox site to another and thus contributes to an intersite flux. This contribution is indicated by the color of the arrows, where black indicates electron transfer between the SP and heme $c_{559}$, blue indicates transfer between heme $c_{559}$ and heme $c_{552}$, red indicates transfer between heme $c_{552}$ and heme $c_{556}$, and green indicates transfer between heme $c_{556}$ and heme $\mathrm{c}_{554}$.

The first simulation, Figure 6a, already gives an example of the next-neighbor character of electron transfer between the four hemes and the SP. Initially, all four hemes are reduced, and the SP is oxidized. Re-reduction of the SP, as known from experiments, occurs via an electron transfer from heme $\mathrm{c}_{559}$, i.e., via the microstate transition $(0,1,1,1,1) \rightarrow(1,0,1,1,1)$. This initial transfer is rapidly followed by an electron transfer from heme $c_{552}$ to heme $c_{559}((1,0,1,1,1) \rightarrow(1,1,0,1,1))$. The necessary intermediate state $(1,0,1,1,1)$ is only transiently populated (maximum $\approx 0.01 \%$ ) and could thus not be observed experimentally. Further relaxation of the system toward its equilibrium distribution again follows the next-neighbor rule. A direct electron transfer from heme $\mathrm{c}_{554}$ to heme $\mathrm{c}_{552}$, although 
States

a)

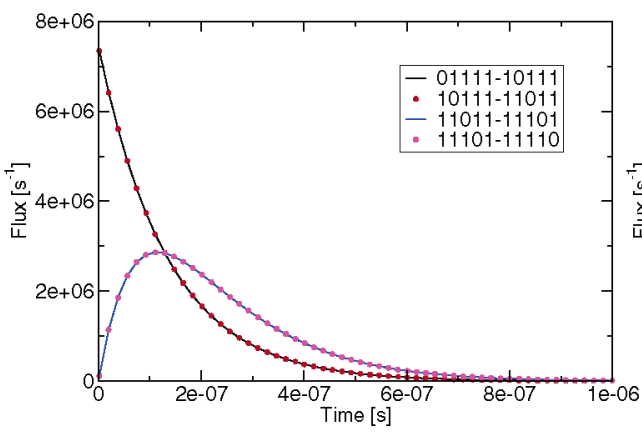

b)

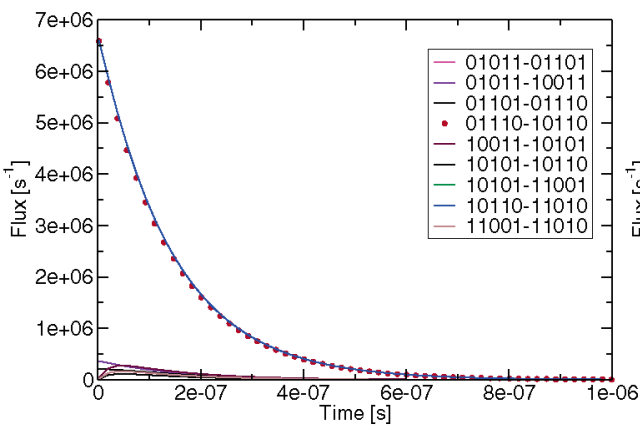

c)

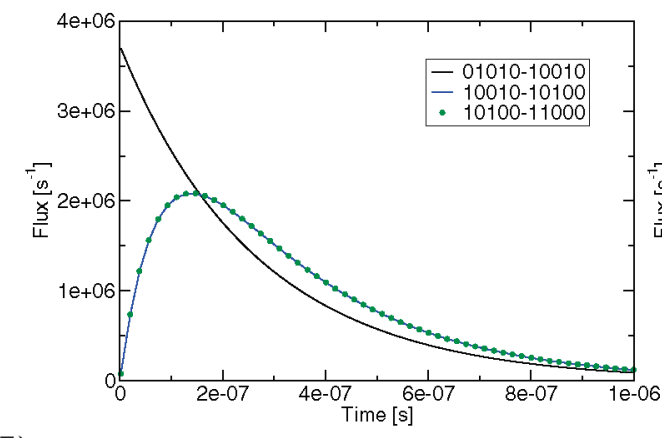

d)

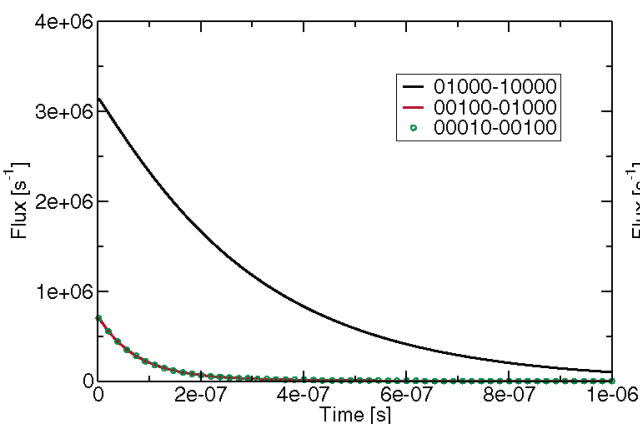

Sites

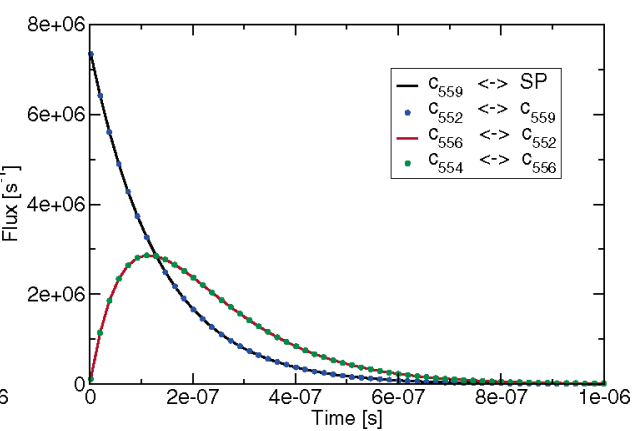

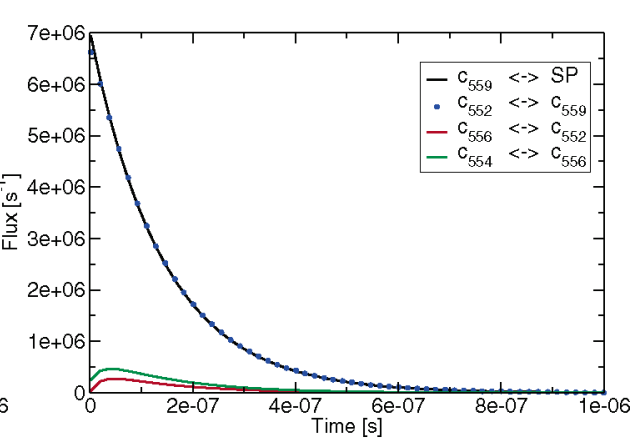

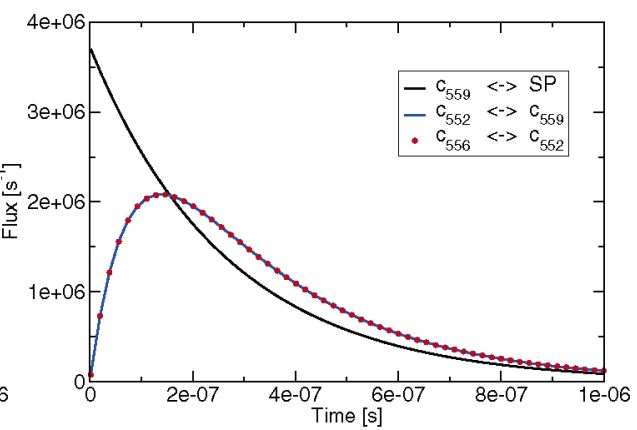



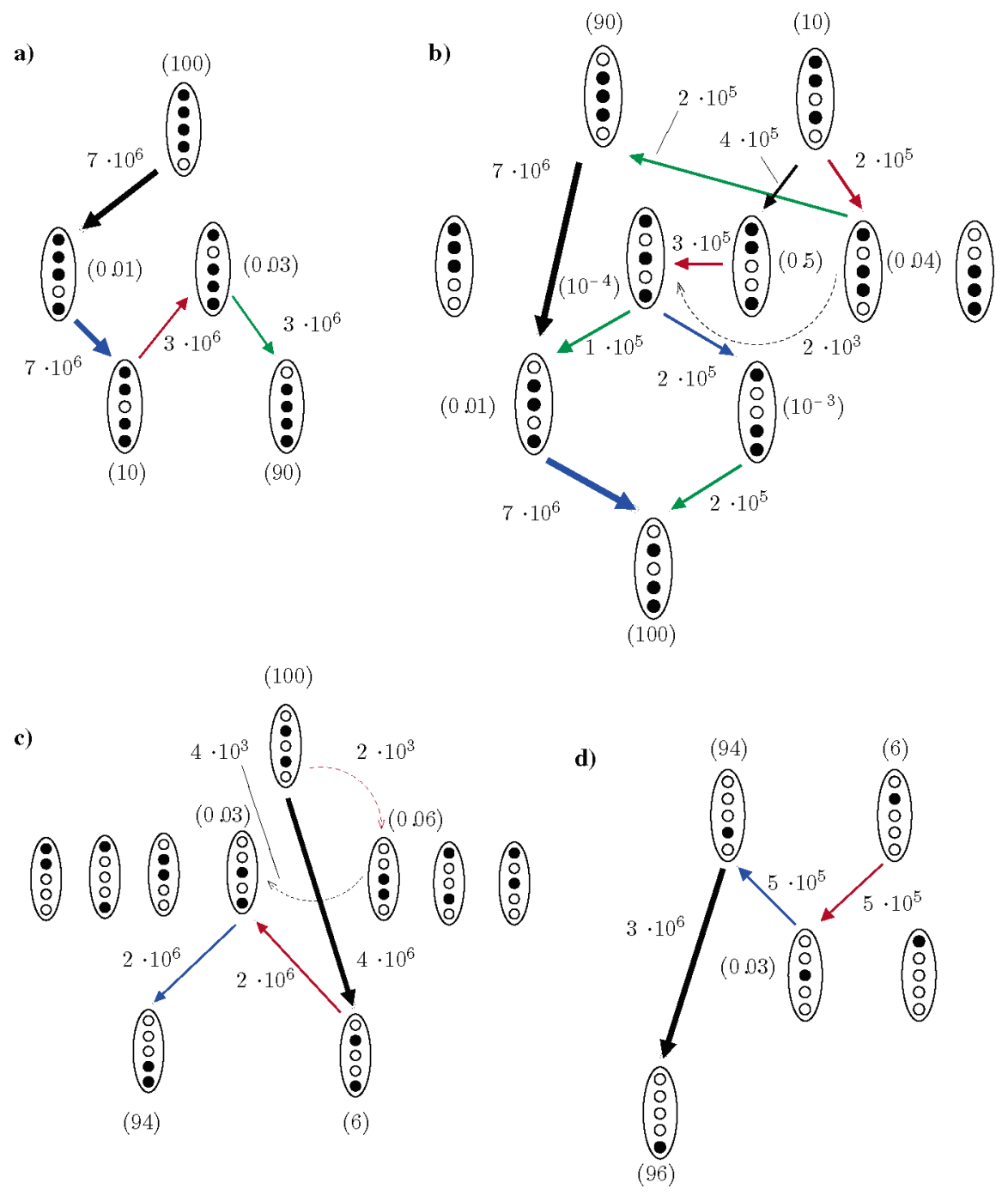

Figure 6. Reaction scheme for the charge transfer in the RC as deduced from the flux analysis of our simulations. Each oval represents a microstate of the system. The circles symbolize the redox cofactors in the order heme $\mathrm{c}_{554}$, heme $\mathrm{c}_{556}$, heme $\mathrm{c}_{552}$, heme $\mathrm{c}_{559}$, and SP from the top to the bottom. Filled and open circles denote the reduced and oxidized forms of the sites, respectively. Panels $\mathrm{a}-\mathrm{d}$ describe the reaction scheme after the first to fourth flash, respectively. Each has a particular order: Initial states are on top, intermediate states are in the middle, and final states are at the bottom. The initial, final, and maximum probabilities are given in parenthesis for initial, final, and intermediate microstates, respectively. Interstate fluxes significantly contributing to the kinetic behavior are indicated by arrows. The associated intersite transfer is indicated by color: Black indicates transfer between heme $c_{559}$ and the SP, blue between heme $c_{552}$ and heme $c_{559}$, red between heme $c_{556}$ and heme $c_{552}$, and green between heme $\mathrm{c}_{554}$ and heme $\mathrm{c}_{556}$.

rapidly decay into the final state via just one intermediate, $(1,0,1,1,0)$. In contrast, the initial state $(0,1,0,1,1)$ has to relax toward the final state via a succession of several intermediates due to the next-neighbor restriction discussed already. Again, these intermediate states are only transiently populated. Each interstate flux into one of these intermediates is accompanied by an equally high flux out of these intermediates. For example, the transition from the initial state to the intermediate $(1,0,0,1,1)$ is rapidly followed by a transition to a second intermediate state $(1,0,1,0,1)$. This intermediate state in turn decays either into state $(1,0,1,1,0)$ via an electron transfer from heme $c_{554}$ to heme $\mathrm{c}_{556}$ or alternatively to state $(1,1,0,0,1)$ via electron transfer from heme $c_{552}$ to heme $c_{559}$. As can be seen from the arrow colors in Figure 6b, electron transfer between two given sites is represented by more than one interstate transition. This multiplicity is the main obstacle in defining rate constants for transfer reactions between two given sites. The presented microstate formalism, however, naturally incorporates this multiplicity and thus avoids the common ambiguities.

The experimental setup resembled in the last two simulations $c$ and d, i.e., transfer with two or one reduced cofactors present in the system, has been widely discussed in the context of uphill electron transfer. ${ }^{10,28,63}$ In Figure 4c, for example, it can be seen that the reduction of the SP is accompanied by the (partial) oxidation of heme $c_{559}$ and heme $c_{556}$ whereas heme $c_{552}$ remains basically oxidized throughout. This behavior, however, must not be taken as evidence for electron transfer between heme $\mathrm{c}_{556}$ and heme $\mathrm{c}_{559}$ or even between heme $\mathrm{c}_{556}$ and the SP. Again, the distances between heme $c_{556}$ and heme $c_{559}(\sim 22 \AA)$ and between heme $c_{556}$ and the SP $(\sim 37 \AA)$ render electron-transfer rates based on eq 11 too slow to contribute significantly to the re-reduction kinetics of the SP. This result is in agreement with mutational studies on the RC of $B$. viridis that suggested that electron transfer always occurs along the sequence $c_{556}-c_{552}-$ $\mathrm{c}_{559}-\mathrm{SP} .{ }^{28}$ Thus, oxidation of heme $\mathrm{c}_{556}$ should be accompanied by reduction of heme $c_{552}$ although heme $c_{552}$ is observed to remain basically oxidized throughout the simulation. This apparent contradiction arising from the experimentally accessible data can be readily resolved in the microstate formalism. As discussed already, only next-neighbor electron-transfer contributes to the transitions in our simulations due to the strong distance dependence in the rate formula (eq 11). The intermedi- 
ate states showing a reduced heme $\mathrm{c}_{552}$, however, are only transiently populated. The maximal probabilities for the intermediates in the simulations of Figure $4 \mathrm{c}$ and $4 \mathrm{~d}$ amount to $\sim 0.02 \%$ for states $(1,0,1,0,0)$ and $(0,0,1,0,0)$, and thus, reduction of heme $c_{552}$ is hardly accessible to experimental observation in these kinetic experiments.

\section{Conclusions}

In the present article, we present a microstate formalism to simulate charge-transfer systems. The system is described by state vectors, indicating the redox form of each site possibly taking part in the transfer process. In this approach, charge transfer is not primarily described as a transfer of a charge from one site to another but as a change in the state vector of the complete system. Thus, there are as many possible transfer reactions between two given sites as there are possible configurations of all other sites. A microstate formalism naturally takes into account the dependence of transfer rates between sites on their interaction with the surrounding charges. For the interconversion between two microstates, a unique chargetransfer rate can be defined. This formalism allows us to describe the kinetics of a charge-transfer system by a master equation describing the time evolution of microstates. The possibly complex kinetic behavior of single sites of the system can be deduced from the time-dependent probability distribution of microstates. Charge flow within the system can be investigated by calculating elementary fluxes between microstates and relating them to the accompanied charge transfer between sites. Thus, the transfer network can be built up by elementary fluxes. Poisson-Boltzmann electrostatics together with existing heuristic rate laws ${ }^{6,8}$ can be used to describe biological electrontransfer systems. The overall kinetics of the system are simulated using coupled differential equations. In this work, the rereduction of the SP by the $\mathrm{C}$-subunit of the $\mathrm{RC}$ serves as a model system to compare the simulations with experiments. All parameters required for calculating electron-transfer rates can be obtained from calculations using structural data. It is therefore possible to simulate the kinetics of an electron-transfer system solely based on structural information without referring to additional experimental data. Our simulations reproduce the experimentally observed re-reduction kinetics of the SP. The calculated lifetimes of the photo-oxidized state of the SP agree well with the experimentally determined values.

All calculations put together in this article to obtain a coherent scheme for simulating the kinetics of an electron-transfer system use standard computational techniques that have been demonstrated to reproduce experimental results for a broad range of biological electron-transfer systems. The formalism presented here should, thus, be readily applicable to a large class of related charge-transfer systems.

Acknowledgment. This work was supported by the Deutsche Forschungsgemeinschaft (Grant No. UL 174/7-1). We thank Donald Bashford for providing his program MEAD and useful discussions. R.T.U. thanks the Universität Bayern e.V. for a fellowship.

\section{References and Notes}

(1) Nicholls, D. G.; Ferguson, S. J. Bioenergetics 3; Academic Press: New York, 2002.

(2) Saraste, M. Science 1999, 283, 1488-1493.

(3) Lancaster, C. R. D.; Michel, H. In Handbook of Metalloproteins; Albrecht Messerschmidt, A., Huber, R., Poulos, T., Wieghardt, K., Eds.; Wiley: New York, 2001; pp 119-135.
(4) Marcus, R. A. J. Chem. Phys. 1956, 24, 966-978.

(5) Marcus, R. A. J. Chem. Phys. 1956, 24, 979-989.

(6) Moser, C. C.; Keske, J. M.; Warncke, K.; Farid, R. S.; Dutton, P. L. Nature 1992, 355, 796-802.

(7) Moser, C. C.; Page, C. C.; Chen, X.; Dutton, P. L. J. Biol. Inorg. Chem. 1997, 2, 393-398.

(8) Page, C. C.; Moser, C. C.; Chen, X.; Dutton, P. L. Nature 1999 402, 47-52.

(9) Page, C. C.; Moser, C. C.; Dutton, P. L. Curr. Opin. Chem. Biol. 2003, 7, 551-556.

(10) Alric, J.; Lavergne, J.; Rappaport, F.; Verméglio, A.; Matsuura, K.; Shimada, K.; Nagashima, K. V. J. Am. Chem. Soc. 2006, 128, 41364145 .

(11) Noy, D.; Moser, C. C.; Dutton, P. L. Biophys. Biochem. Acta 2006, 1757, 90-105.

(12) Bashford, D.; Gerwert, K. J. Mol. Biol. 1992, 224, 473-486

(13) Ullmann, G. M.; Knapp, E.-W. Eur. Biophys. J. 1999, 28, 533551 .

(14) Sharp, K. E. Biophys. J. 1998, 73, 1241-1250.

(15) Onufriev, A.; Case, D. A.; Ullmann, G. M. Biochemistry 2001, $40,3413-3419$.

(16) Ullmann, G. M. J. Phys. Chem. B 2003, 107, 1263-1271.

(17) Alric, J.; Cuni, A.; Maki, H.; Nagashima, K. V.; Verméglio, A.; Rappaport, F. J. Biol. Chem. 2004, 279, 47849-47855.

(18) Sham, Y. Y.; Muegge, I.; Warshel, A. Proteins 1999, 36, 484500.

(19) Nogi, T.; Hirano, Y.; Miki, K. Photosynth. Res. 2005, 85, 87-99.

(20) Deisenhofer, J.; Epp, O.; Miki, K.; Huber, R.; Michel, H. J. Mol. Biol. 1984, 180, 358-398.

(21) Deisenhofer, J.; Epp, O.; Miki, K.; Huber, R.; Michel, H. Nature 1985, 318, 618-624.

(22) Deisenhofer, J.; Epp, O.; Sinning, I.; Michel, H. J. Mol. Biol. 1995, 246, 429-457.

(23) Dracheva, S. M.; Drachev, L. A.; Konstantinov, A. A.; Semenov, A. Y.; Skulachev, V. P.; Arutjunjan, A. M.; Shuvalov, V. A.; Zaberezhnaya, S. N. Eur. J. Biochem. 1988, 171, 253-264.

(24) Fritzsch, G.; Buchanan, S.; Michel, H. Biochim. Biophys. Acta 1989, 977, 157-162.

(25) Ortega, J.; Mathis, P. Biochemistry 1993, 32, 1141-1151.

(26) Rappaport, F.; Béal, D.; Verméglio, A.; Joliot, P. Photosynth. Res 1998, 55, 317-323.

(27) Ortega, J.; Drepper, F.; Mathis, P. Photosynth. Res. 1999, 59, 147157.

(28) Chen, I.-P.; Mathis, P.; Koepke, J.; Michel, H. Biochemistry 2000, $39,3502-3602$.

(29) Gunner, M.; Honig, B. Proc. Natl. Acad. Sci. U.S.A. 1991, 88, 9151-9155

(30) Scharnagl, C.; Hettenkofer, J.; Fischer, S. F. Int. J. Quantum Chem. 1994, 52, 33-56.

(31) Onufriev, A.; Smondyrev, A.; Bashford, D. J. Mol. Biol. 2003, 332, $1183-1193$

(32) Ferreira, A.; Bashford, D. J. Am. Chem. Soc. 2006, 128, 1677816790

(33) Ullmann, G. M. J. Phys. Chem. B 2000, 104, 6293-6301.

(34) Marcus, R. J. Chem. Phys. 1963, 38, 1858-1862.

(35) Marcus, R. A.; Sutin, N. Biochim. Biophys. Acta 1985, 811, 265322.

(36) Williams, R. J. P. J. Solid State Chem. 1999, 145, 488-495.

(37) Olsson, M. H. M.; Ryde, U.; Roos, B. O. Protein Sci. 1998, 81, 6554-6558.

(38) Ryde, U.; Olsson, M. H. M. Int. J. Quantum Chem. 2001, 81, 335347

(39) Higuchi, Y.; Kusunoki, M.; Matsuura, N.; Yasuoka, N.; Kakudo, M. J. Mol. Biol. 1984, 172, 109-139.

(40) Brünger, A. T.; Karplus, M. Proteins 1988, 4, 148-156.

(41) Brooks, B. R.; Bruccoleri, R. E.; Olafson, B. D.; States, D. J.; Swaminathan, S.; Karplus, M. J. Comput. Chem. 1983, 4, 187-217.

(42) MacKerell, A. D.; Bashford, D.; Bellott, M.; Dunbrack, R. L., Jr ; Evanseck, J. D.; Field, M. J.; Fischer, S.; Gao, J.; Guo, H.; Ha, S.; JosephMcCarthy, D.; Kuchnir, L.; Kuczera, K.; Lau, F. T. K.; Mattos, C.; Michnick, S.; Ngo, T.; Nguyen, D. T.; Prodhom, B.; Reiher, W. E., III.; Roux, B.; Schlenkrich, M.; Smith, J. C.; Stote, R.; Straub, J.; Watanabe, M.; Wiorkiewicz-Kuczera, J.; Yin, D.; Karplus, M. J. Phys. Chem. B 1998 102, 3586-3616.

(43) Vosko, S. H.; Wilk, L.; Nusair, M. Can. J. Phys. 1980, 1200, 58.

(44) Perdew, P.; Chevary, J. A.; Vosko, S. H.; Jackson, K. A.; Pederson, M. R.; Singh, D. J.; Fiolhais, C. Phys. Rev. B 1992, 46, 6671.

(45) Guerra, C. F.; Snijders, J. G.; te Velde, G.; Baerends, E. J. Theor. Chem. Acta 1998, 391, 391-403.

(46) Breneman, C. N.; Wiberg, K. B. J. Comput. Chem. 1990, 11 (3), $361-373$. 
(47) Chen, J. L.; Noodleman, L.; Case, D.; Bashford, D. J. Phys. Chem. 1994, 98, 11059-11068.

(48) Rabenstein, B.; Ullmann, G. M.; Knapp, E. W. Biochemistry 1998 , $37,2488-2495$

(49) Rabenstein, B.; Ullmann, G. M.; Knapp, E. W. Biochemistry 2000 , $39,10487-10496$

(50) Taly, A.; Sebban, P.; Smith, J. C.; Ullmann, G. M. Biophys. J. 2003, 84, 2090-2098.

(51) Bashford, D.; Case, D. A.; Dalvit, C.; Tennant, L.; Wright, P. E. Biochemistry 1993, 32, 8045-8056.

(52) Ullmann, G. M.; Knapp, E. W. Eur. Biophys. J. 1999, 28, 533551.

(53) Becke, A. D. Phys. Rev. A 1988, 38, 3098-3100

(54) Ryde, U.; Olsson, M. H. M.; Roos, B. O.; Borin, C. A. Theor Chem. Acc. 2001, 105, 452-462.

(55) Oppenheim, I.; Shuler, K. E.; Weiss, G. H. Stochastic Processes in Chemical Physics: The Master Equation; MIT Press: Cambridge, MA, 1977.
(56) van Kampen, N. Stochastic Processes in Physics and Chemistry; North-Holland: New York, 1981.

(57) Anderson, E.; Bai, Z.; Bischof, C.; Blackford, S.; Demmel, J.; Dongarra, J.; Du Croz, J.; Greenbaum, A.; Hammarling, S.; McKenney, A.; Sorensen, D. LAPACK Users' Guide, 3rd ed.; Society for Industrial and Applied Mathematics: Philadelphia, PA, 1999.

(58) Press, W.; Teukolsky, S.; Vetterling, W.; Flannery, B. Numerical Recipes in $C$, 2nd ed.; Cambridge University Press: New York, 1992.

(59) Voigt, P.; Knapp, E.-W. J. Biol. Chem. 2003, 278, 5199352001.

(60) Muegge, I.; Qi, P.; Wand, A.; Chu, Z.; Warshel, A. J. Phys. Chem. $B$ 1997, 101 (5), 825-836.

(61) Parson, W.; Chu, Z.; Warshel, A. Biophys. J. 1998, 74 (1), 182191.

(62) Mertz, E. L.; Krishtalik, L. I. Proc. Natl. Acad. Sci. U.S.A. 2000, 97, 2081-2086

(63) Nogi, T.; Miki, K. J. Biochem. 2001, 130, 319-329. 